\title{
Stony the road we trod: towards racial justice in kidney care
}

\section{O. N. Ray Bignall Il and Deidra C. Crews}

\section{The COVID-19 pandemic and the Movement for Black Lives have focused attention on racial disparities in kidney health outcomes. In 2020, kidney professionals highlighted threats posed by racism and other negative social drivers of kidney health, and proposed solutions to address these issues through scholarship and advocacy for social justice.}

Racism is one of America's most enduring public health risks. Past-president of the American Public Health Association, Camara Jones defines racism as a "system of structuring opportunity and assigning value based on the social interpretation of how one looks (which is what we call 'race'), that unfairly disadvantages some individuals and communities, unfairly advantages other individuals and communities, and saps the strength of the

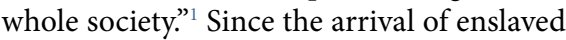
Africans in 1619, Black individuals have encountered racist systems that preclude their full participation in America's scientific, economic and social progress. In 2020, the killings of Ahmaud Arbery, Breonna Taylor, George Floyd and others focused fresh attention on the scourge of racism.

The practice and academy of medicine are not immune to the effects of racism. Historically, racist pseudoscience has been used to justify the unequal treatment of Black people. Today, racial bias in society is reflected in health care, resulting in the disparate health outcomes experienced by Black patients. The collision of the COVID-19 pandemic with high-profile instances of racial injustice and violence has revealed the unique vulnerability of Black communities to these threats. On one hand, Black Americans are at increased risk of reflected in health care
SARS-CoV-2 infection owing to a legacy of residential segregation and economic immobility that forces many into low wage 'essential work' and limits their access to timely, quality health care. These factors contribute to wide disparities in COVID-19 morbidity and mortality, including a $23 \%$ increased risk of acute kidney injury among Black Americans with COVID $-19^{2}$. On the other hand, the cumulative effects of repeated exposure of Black Americans to experiences of racial discrimination contributes to a weathering phenomenon that exacerbates adverse health outcomes ${ }^{3}$.

In response to these issues, thousands of kidney professionals marched, knelt and demonstrated for racial justice in 2020. Many are asking "what should we do now?" In the past year, members of the kidney community have informed the way forward to address these challenges.

First, kidney professionals must be willing to stand for social justice. This process begins by acknowledging the reality of systemic inequities, including systemic racism and recognizing that advancing equity in society can move us closer to equity in kidney health. Among patients with COVID-19, kidney health disparities are linked to inequities in social drivers of health, such as access to health care, employment, housing and the environment ${ }^{4}$. The kidney community must seek greater knowledge about the lived experiences of people who face these challenges. Advocating and voting for policies that reduce housing instability, rectify economic kidney professionals must be willing to stand for social justice

inequality, and promote equitable access to nutrient-rich foods and clean water could dismantle barriers for patients and families living with chronic kidney disease (CKD).

Second, kidney professionals must recognize the impact of systemic inequities on kidney health in their scholarship and clinical practice. Systemic bias and social factors adversely impact kidney care in many communities. In 2020, an analysis of data from the Systolic Blood Pressure Intervention Trial found no differences in blood pressure trajectory, number of medications needed to achieve target blood pressure, trends in kidney function or left ventricular mass between Black participants with increasing tertiles of West African ancestry ${ }^{5}$. Rather than a genetic basis for racial disparities in cardiovascular risk, this finding suggests a role for structural, societal drivers ${ }^{5}$. Moreover, a study of Black men with kidney failure found that those who received dialysis in communities with higher proportions of Black residents had a significantly increased risk of hospitalization, suggesting an influence of neighborhood-level disparities on clinical outcomes in this group ${ }^{6}$. Systemic obstacles also reduce access of Black Americans to preemptive kidney transplantation. Potential approaches to combat this problem include addressing low kidney health literacy, insurance barriers and the inconsistent use of estimated glomerular filtration rate (eGFR) cutpoints in transplant waitlisting criteria ${ }^{7}$. Indeed, the American Society of

Lift Every Voice and Sing (2nd verse)

Stony the road we trod,

Bitter the chastening rod,

Felt in the days when hope unborn had died;

Yet with a steady beat,

Have not our weary feet

Come to the place for which our fathers sighed?

We have come over a way that with tears has been watered,

We have come, treading our path through the blood of the slaughtered,

Out from the gloomy past,

Till now we stand at last

Where the white gleam of our bright star is cast.

James Weldon Johnson, 1900 


Key advances
- Racism is a public health crisis that
underlies the adverse health outcomes
experienced by Black Americans, including
in the settings of kidney disease and
COVID-19.
- Kidney professionals must take a stand for
social justice by acknowledging the reality
of systemic inequities and systemic racism,
recognizing that advancing equity in
society can move us closer to kidney health
equity for all'.
- The impact of systemic inequities on kidney
health must be addressed by kidney
professionals in their scholarship and
clinical practice; systemic bias and social
drivers can negatively impact kidney care,
especially within communities that are
adversely affected by health disparaties ${ }^{4}$.
- Championing workforce diversity and
inclusion must be a priority for the kidney
community; a diverse and inclusive
workforce drives clinical excellence,
therapeutic rapport and discovery.
- The development of robust community
engagement, partnership, and leadership is
necessary to build trust within underserved
and minority populations that historically
have been victimized by acts of injustice
in medicine ${ }^{10}$.

Nephrology (ASN) and the National Kidney Foundation are currently reassessing the inclusion of race in eGFR equations with the aim of ensuring that $\mathrm{CKD}$ diagnostic methodologies do not contribute to health disparities ${ }^{4}$.

Third, championing workforce diversity and inclusion should be a priority for the kidney community. A consensus is growing that acknowledges a diverse and inclusive workforce as a key driver of clinical excellence, therapeutic rapport and discovery. Unfortunately, the diversity of the nephrology workforce is failing to match that of patients with kidney disease. In 2020, Lederer and Lebowitz noted that progress towards diversity in nephrology has been slow, and recommended that specific efforts be directed towards increasing racial, ethnic and gender representation $^{8}$. These efforts include supporting the advancement of women in nephrology and the elimination of 'manels' (all male scholarly panels) at academic conferences. In addition, focused initiatives such as the ASN Loan Mitigation Program aim to increase representation of immigrant communities and groups who are currently underrepresented in medicine, including Black, Latinx and Indigenous people. Olayiwola and Choo argued that health-care organizations should be held accountable for the creation of safe, supportive, and inclusive environments for training, scholarship and clinical care?

\section{a diverse and inclusive workforce as a key driver of clinical excellence, therapeutic rapport and discovery}

Finally, there is an urgent need to build trust through authentic community collaboration. The written and oral history of Black Americans is replete with episodes of medical experimentation, exploitation and neglect, old and new. This history includes the victimization of enslaved women like Anarcha Westcott and the abused men of the Tuskegee Syphilis Study as well as harrowing accounts of medical neglect experienced by Black women in childbirth. These accounts include the recent experiences of the tennis icon Serena Williams who developed life-threatening complications following an emergency C-section, and the late epidemiologist Shalon Irving who tragically died due to complications of high blood pressure 3 weeks after giving birth. Black Americans know these stories well, and many have personal anecdotes of perceived disregard and/or mistreatment by the medical system. This accumulated knowledge creates an iatrophobia that impedes participation of Black Americans in clinical trials and immunization programs as well as their access to novel therapies. In the words of Kimberly Manning writing about her experience as a Black American physician enrolling in a clinical trial, "we are not simply untrusting - we remember ${ }^{10}$." Efforts to remove these barriers begin with an acknowledgement of this painful history and continue with the development of community partnerships that leverage shared leadership to achieve kidney health equity for all Americans.

In 2020, the academic kidney community shed light on the stony road we've trod to acknowledge deep racial disparities in kidney disease. We must now use our collective scholarship and expertize to put kidney health equity into action.

O. N. Ray Bignall II ${ }^{1 凶}$ and Deidra C. Crews ${ }^{2,3}$

${ }^{1}$ Division of Nephrology and Hypertension, Department of Pediatrics, Nationwide Children's Hospital and The Ohio State University College of Medicine, Columbus, $\mathrm{OH}$, USA.

2Division of Nephrology, Department of Medicine, Johns Hopkins University School of Medicine, Baltimore, MD, USA.

${ }^{3}$ Johns Hopkins Center for Health Equity and Welch Center for Prevention, Epidemiology and Clinical Research, Johns Hopkins Medical Institutions, Baltimore, MD, USA.

凶e-mail: ray.bignall@nationwidechildrens.org https://doi.org/10.1038/s41581-020-00389-w

1. Jones, C. P. Toward the science and practice of anti-racism: launching a National Campaign against Racism. Ethnicity \& disease 28, 231-234 (2018).

2. Hirsch, J. S. et al. Acute kidney injury in patients hospitalized with COVID-19. Kidney Int. 98, 209-218 (2020).

3. Crews, D. C. \& Purnell, T. S. COVID-19, racism, and racial disparities in kidney disease: galvanizing the kidney community response. J. Am. Soc. Nephrol. 31 1-3 (2020).

4. Longino, K. \& Kramer, H. Racial and ethnic disparities, kidney disease, and COVID-19: a call to action. Kidney Med. 2, 509-510 (2020).

5. Rao, S. et al. Association of genetic West African ancestry, blood pressure response to therapy, and cardiovascular risk among self-reported Black individuals in the Systolic Blood Pressure Reduction Intervention Trial (SPRINT). JAMA Cardiology https:// doi.org/10.1001/jamacardio.2020.6566 (2020).

6. Golestaneh, L. et al. The role of place in disparities affecting Black men receiving hemodialysis. Kidney Int. Rep. https://doi.org/10.1016/j.ekir.2020.10.014 (2020).

7. Reese, P. P. et al. Racial disparities in preemptive waitlisting and deceased donor kidney transplantation: ethics and solutions. Am. J. Transplant. https://doi.org/ 10.1111/ajt.16392 (2020).

8. Lederer, E. \& Lebowitz, J. Current state of the workforce in nephrology. Adv. Chronic Kidney Dis. 27, 281-290 (2020)

9. Olayiwola, J. N. \& Choo, E. Seven more things organisations should be doing to combat racism. Lancet 396, 593 (2020).

10. Manning, K. D. More than medical mistrust. Lancet 396, 1481-1482 (2020)

Competing interests

The authors declare no competing interests. 